\title{
Model-Based Estimation of Electrocardiographic QT Interval From Phonocardiographic Heart Sounds in Healthy Subjects
}

\author{
Agnese Sbrollini ${ }^{1}$, Micaela Morettini ${ }^{1}$, Ilaria Marcantoni ${ }^{1}$, Laura Burattini ${ }^{*}{ }^{, 1}$ \\ ${ }^{1}$ Università Politecnica delle Marche, Ancona, Italy
}

\begin{abstract}
The electrocardiographic $Q T$ interval is an index of cardiac risk commonly used in clinics. Accurate $Q T$ measure is challenging, especially in noisy conditions, when acquisitions of phonocardiograms (PCGs) may be more reliable than acquisitions of electrocardiograms (ECGs). However, PCG features are less used in clinics. Thus, aim of the study was to propose a model for indirectly measuring the electrocardiographic QT interval from the phonocardiographic heart sounds in healthy subjects. To this aim, simultaneously acquired PCGs and ECGs of 99 healthy subjects were processed to obtain median PCG and ECG beats. Beat length, S1 onset and S2 onset were identified from the median PCG beat, while QT interval $(Q T)$ was measured from the median ECG beat. Then, a regression model was formulated by regression analysis to obtain PCG-based QT estimation $(\widehat{Q T})$ and validated by leave-one-out cross-validation. Correlation coefficient $(\rho)$ and estimation error were also computed. $\widehat{Q T}$ and QT did not differ significantly (model formulation: $362 \mathrm{~ms}$ vs 358ms; model validation:360ms vs 358ms, respectively; $P>0.5)$ and were significantly correlated (model formulation: $\rho=0.7, P<10^{-13}$; model validation: $\left.\rho=0.6, P<10^{-10}\right)$; median error is $1 \mathrm{~ms}(<0.5$ in \%). Thus, the proposed model provides a reliable estimation of $Q T$ interval from PCG heart sounds in healthy subjects.
\end{abstract}

\section{Introduction}

Cardiac monitoring is frequently performed though the electrocardiogram (ECG), which is the recording of the electrical activity of the cardiac cells typically by skin electrodes located in standardized positions. In particular, the electrocardiographic QT interval is a common, clinically used index to assess cardiac electrical risk in clinical trials [1-3]. The QT interval is defined as the time interval between the QRS-complex onset and the T-wave offset [4], thus representing duration of both ventricular depolarization (represented by the QRS complex) and repolarization (represented by the $\mathrm{T}$ wave). Due to the smoothness of the electrocardiographic waves and the difficulties to correctly identify their onsets and offsets, accurate QT-interval measurement remains challenging [5].

Beside through ECG, cardiac monitoring can also be performed through the phonocardiogram (PCG), which is the recording of the heart sounds generated by the closure of the cardiac atrioventricular and aortic-pulmonary valves. Typically, PCG is acquired by stethoscope, but can also be obtained by acoustic sensors on the chest, as it occurs in wearable sensors [6]. In the PCG, the closure of the atrioventricular valves is represented by the first heart sound (S1) and the closure of the aortic-pulmonary valves is represented by the second heart sound (S2). Physiologically, S1 represents the beginning of ventricular systole and S2 represents the beginning of ventricular diastole [4].

In healthy conditions, the electrical activity of heart drives its mechanical activity that directly causes the valves closure [4]. Thus, ECG and PCG are strictly related, as it can be easily observed by comparing two simultaneously acquired ECG and PCG recordings (Figure 1). Obviously, both recordings contain the same number of cardiac cycles, since they are different representations of the same cardiac activity. Additionally, QRS complexes appear to be aligned with $\mathrm{S} 1$ occurrences and $\mathrm{T}$ waves offset appear to be close in time to S2 onset (a previous study of ours indicate that they are, on average, $5 \mathrm{~ms}$ apart [7]).

Despite electrocardiography and phonocardiography being both noninvasive methods for cardiac monitoring [8], to acquire a PCG recording is usually much easier than to acquire an ECG recording, and PCG features identification is less sensitive to noise corruption. These characteristics make PCG recordings more reliable than ECG recordings, especially when obtained using wearable sensors [6] that typically provide recordings affected by high levels of noise. On the other hand, ECG features, such the popular QT interval, are much more used in clinics that PCG features.

By considering the facility of PCG recording, its analogies with ECG and the clinical significance of the QT interval, aim of the present study was to propose a model for electrocardiographic QT-interval estimation from phonocardiographic heart sounds in healthy subjects. 


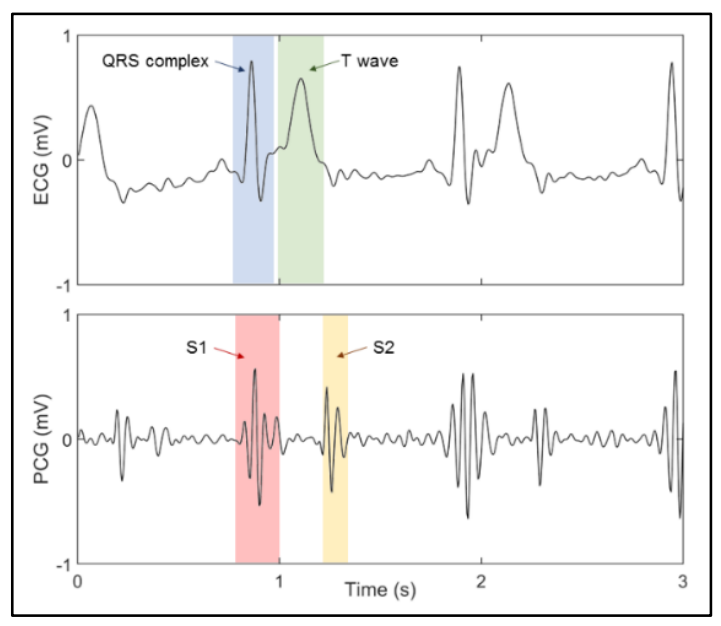

Figure 1. Waveform correspondence in simultaneously acquired ECG and PCG recordings.

\section{Material and Methods}

\subsection{Data}

Data considered in this study belong to the training set A of PhysioNet/CinC Challenge 2016 database [9,10], consisting in 99 pairs of short (around 30s) PCG and ECG signals acquired from healthy subjects. Both signals were simultaneously recorded by the Welch Allyn Meditron electronic stethoscope. ECGs sampling frequency was $1000 \mathrm{~Hz}$, while PCG sampling frequency was $2000 \mathrm{~Hz}$.

\subsection{Preprocessing}

ECG signals were prefiltered using a bidirectional $3^{\text {rd }}$ order Butterworth band-pass filter with $0.5 \mathrm{~Hz}$ and $25 \mathrm{~Hz}$ cut-off frequencies. Additionally, electrocardiographic Rpeak positions were extracted using the Pan-Tompkins algorithm [11]. PCG signals were prefiltered using a bidirectional $3^{\text {rd }}$-order Butterworth low-pass filter with a $20 \mathrm{~Hz}$ cut-off frequency. Both ECG and PCG signals were resampled to $200 \mathrm{~Hz}$ to have the same sampling frequency.

\subsection{Median Cardiac Beats Computation}

Median ECG and median PCG cardiac beats were computed from each pair of simultaneously acquired signals. The electrocardiographic R-peak positions were taken as reference and used to compute the number $\mathrm{N}$ of cardiac beats included in each signals pair and the mean cardiac beat length $(\mathrm{L} ; \mathrm{ms})$. Both cardiac signals were segmented into $\mathrm{N}$ cardiac beats; each beat was identified as the signal segment included between $250 \mathrm{~ms}$ before the R-peak position and L-250ms after the same R-peak position. All cardiac beats segmented on the ECG were used to compute the median ECG beat (MECGB); analogously, all cardiac beats segmented in the PCG were used to compute the median PCG beat (MPCGB).

\section{$2.4 \quad$ Features Extraction}

Four wave landmarks were extracted from the median beats: QRS-complex onset and T-wave offset from MECGB; and S1 onset and S2 onset extracted from MPCGB. QRS-complex onset and T-wave offset were identified by application of the well-known Laguna and Thakor algorithm [12] to MECGB. Instead, S1 onset and S2 onset were identified by application of our thresholdbased algorithm to |MPCGB| [7]. The threshold, defined as twice |MPCGB| standard deviation, crossed |MPCGB| in four points: the onset and the offset of S1, and the onset and the offset of S2. In order to avoid misidentification, time distance between S1 offset and S2 onset had to be smaller than $450 \mathrm{~ms}$ and longer than $60 \mathrm{~ms}$. If this condition was satisfied, S1 onset and S2 onset were confirmed for the study. All four landmarks were visually confirmed by an expert cardiologist.

The following features were computed using the four extracted landmarks (Figure 2): QT (ms), quantified as the time interval from T-wave offset and QRS-complex onset on MECGB; S1on (ms), quantified as the time interval from MPCGB beginning to S1 onset; and S2on (ms), quantified as the time interval from MPCGB beginning to S2 onset.

\subsection{Statistics and Model Construction}

Normality of feature distributions was evaluated by Lilliefors test; non-normal distributions were reported in terms of $50^{\text {th }}$ (median value) $\left[25^{\text {th }} ; 75^{\text {th }}\right]$ percentiles.

In order to obtain a PCG-based QT estimation $(\widehat{\mathrm{QT}})$, S1on, S2on and L were considered as inputs of the regression model reported in Equation 1; QT was considered as reference.

$$
\widehat{\mathrm{QT}}=\mathrm{p}_{1} \cdot \mathrm{S} 1 \text { on }+\mathrm{p}_{2} \cdot \mathrm{S} 2 \text { on }+\mathrm{p}_{3} \cdot \mathrm{L}+\mathrm{p}_{4} \cdot \mathrm{S} 1 \text { on } \cdot \mathrm{S} 2 \text { on }+\mathrm{p}_{5}
$$

where $\mathrm{p}_{1}, \mathrm{p}_{2}$ and $\mathrm{p}_{3}$ are dimensionless, $\mathrm{p}_{4}$ is in $\mathrm{ms}^{-1}$ and $\mathrm{p}_{5}$ is in $\mathrm{ms}$.

The model was formulated by performing a regression analysis between $\widehat{\mathrm{QT}}$ and reference QT interval, in order to identify the numerical values of the five regression coefficients $\mathrm{p}_{1}$ to $\mathrm{p}_{5}$. Moreover, the model was validated by using the leave-one-out cross-validation algorithm [13]. For both model formulation and validation, $\widehat{\mathrm{QT}}$ and QT values were compared using the Wilcoxon ranksum test for equal medians- Additionally, the absolute error $(\varepsilon ; \mathrm{ms})$ and the relative percentage error $(\varepsilon \%)$ between $\widehat{\mathrm{QT}}$ and QT were computed. Finally, the Pearson's correlation analysis was performed by computing the correlation coefficient $(\rho)$ and regression line $(\mathrm{QT}=\mathrm{m} \cdot \widehat{\mathrm{QT}}+\mathrm{q})$ between $\widehat{\mathrm{QT}}$ and $\mathrm{QT}$. 


\section{Results}

Distributions of S1on, S2on and L over the entire dataset were $243[233 ; 250] \mathrm{ms}, \quad 595[575 ; 615] \mathrm{ms}$ and $858[796 ; 961] \mathrm{ms}$, respectively. Values of regression coefficients, obtained with model formulation, were $p_{1}=-$ $3.3, \mathrm{p}_{2}=-0.9, \mathrm{p}_{3}=0.1, \mathrm{p}_{4}=5.4\left(\mathrm{~ms}^{-1}\right)$ and $\mathrm{p}_{5}=825(\mathrm{~ms})$.

Distributions of QT and $\widehat{\mathrm{QT}}$ for both model formulation and validation are reported in Table 1 , together with $\varepsilon$ and $\varepsilon \%$ distributions. In both cases, median $\widehat{\mathrm{QT}}$ value was not statistically different from median QT value $(P=0.61$ and $P=0.84$ for model formulation and validation, respectively). Median $\varepsilon$ values were always $1 \mathrm{~ms}$, whereas median $\varepsilon \%$ was lower than $0.5 \%$ for both model formulation and validation. Eventually, $\widehat{\mathrm{QT}}$ and QT were significantly correlated in both model formulation $(\rho=0.7$; $\left.P<10^{-13}\right)$ and validation $\left(\rho=0.6 ; p<10^{-10}\right)$; regression lines are depicted in Figure 3.

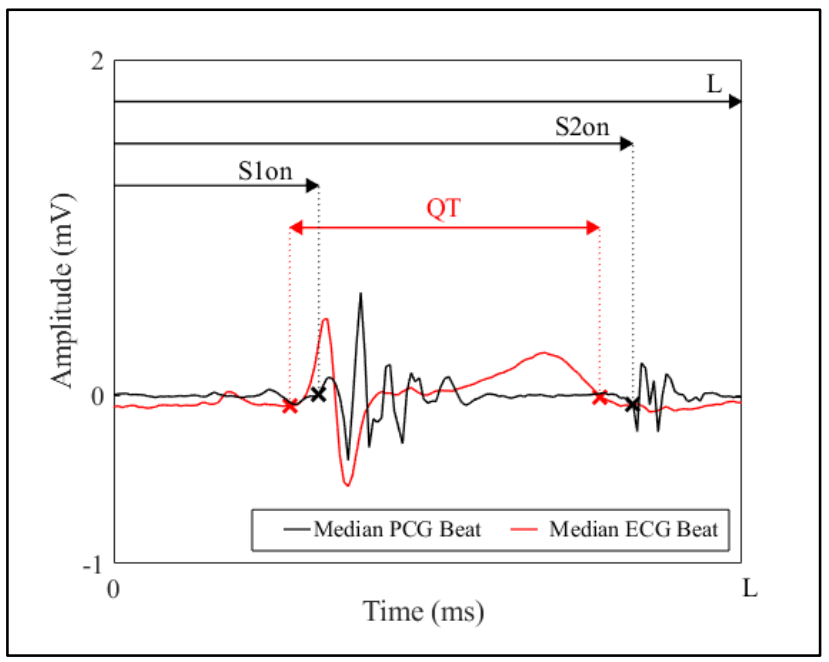

Figure 2. Computation of QT (red double arrow) from the MECGB (in red) and of S1on and S2on (black arrows) from the MPCGB (in black). Red crosses identify QRScomplex onset and T-wave offset; black crosses identify $\mathrm{S} 1$ onset and $\mathrm{S} 2$ onset. $\mathrm{L}$ is the cardiac beats length.

Table 1. Reference QT interval, $\widehat{\mathrm{QT}}$ estimation and errors distributions for both model formulation and validation.

\begin{tabular}{ccc}
\hline & $\begin{array}{c}\text { Model } \\
\text { Formulation }\end{array}$ & $\begin{array}{c}\text { Model } \\
\text { Validation }\end{array}$ \\
\hline QT (ms) & $358[345 ; 377]$ & $358[345 ; 377]$ \\
$\widehat{\text { QT }}(\mathbf{m s})$ & $362[347 ; 373]$ & $360[350 ; 373]$ \\
$\boldsymbol{\varepsilon}(\mathbf{m s})$ & $1[-12 ; 15]$ & $-1[-15 ; 16]$ \\
$\mathbf{\varepsilon \%}$ & $0.02[-0.43 ; 0.52]$ & $-0.16[-3.76 ; 4.64]$ \\
\hline
\end{tabular}

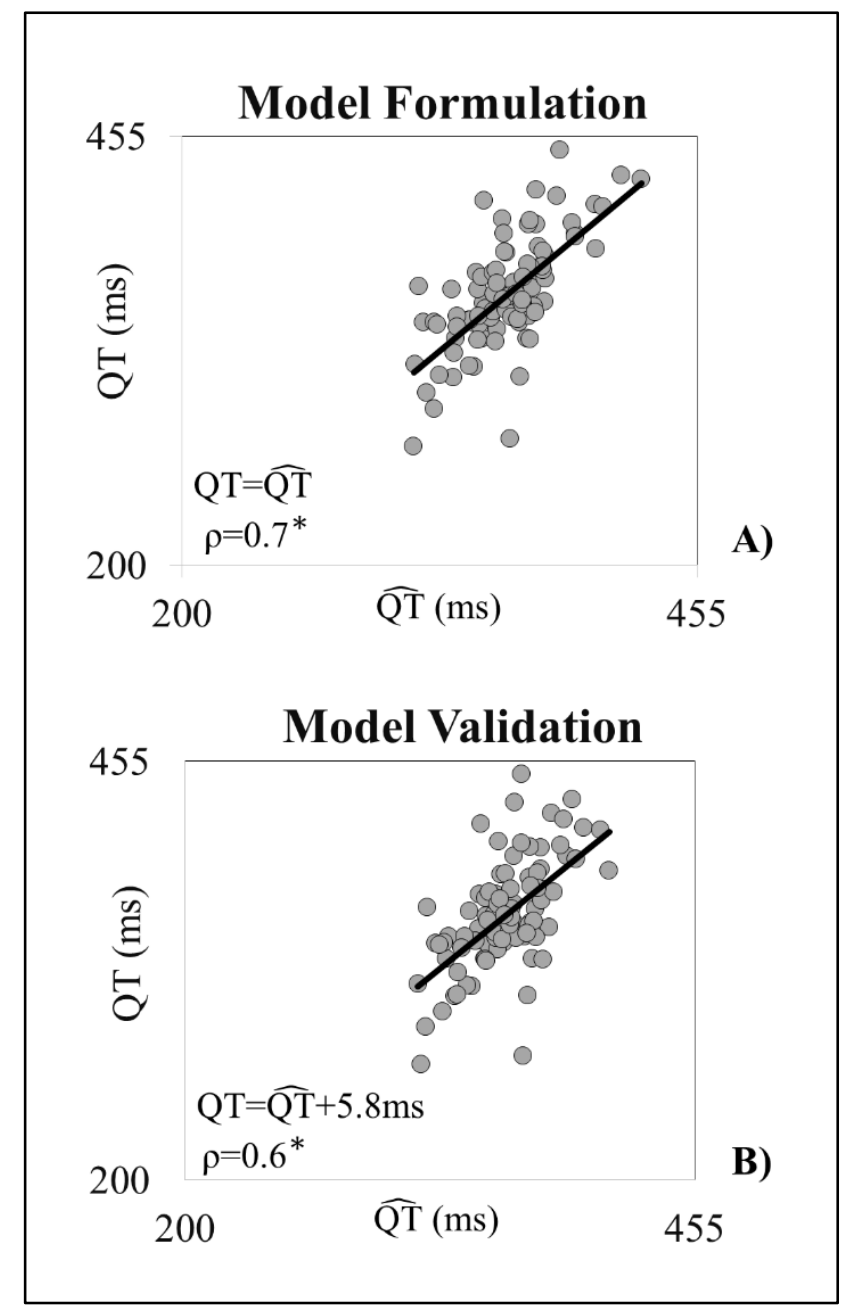

Figure 3. Scatter plots of $\widehat{\mathrm{QT}}$ estimates in relation to QT values (i.e. reference values) for both model formulation (panel A) and validation (panel B). The regression lines are represented in black. Parameters value of regression lines and correlation coefficients are also reported.

\section{Discussion}

This study proposed a model for electrocardiographic QT-interval estimation from phonocardiographic heart sounds in healthy subjects. Indirect measures of the QT interval from PCG is indeed desirable since in several cases, such as during exercise and when using wearable sensors, PCG recordings are more reliable than ECG recordings, but ECG measurements are more popular in clinics.

QT estimation from PCG were obtained by formulating a regression model that uses mean length of the beat and PCG landmarks identifying the beginning of S1 and S2 sound as inputs. The proposed PCG-based model provided 
good results in QT estimation, not statistically different and statistically correlated with the reference QT interval values obtained by directly measuring the QT intervals on the simultaneous ECGs. The small errors between the measured and estimated QT interval (on average $\varepsilon=1 \mathrm{~ms}$; $\varepsilon \%<0.5 \%$ ) is much smaller than the standard variability range (order of $10 \mathrm{~ms}$ ) observed when measuring PCG/ECG landmarks in different electrocardiographic leads, with different methods, and with different levels of noise [5].

Previously, few studies in the literature tried to correlate the PCG features with ECG features [14-17]. These works raised the evidence that the assessment of the link between electrocardiographic QT interval and phonocardiographic features can be promising for the timely diagnosis of coronary artery diseases. Thus, future studies will aim to investigate the possibility to use our PCG/ECG combined approach in the diagnosis of electrical diseases (such as long QT syndrome) or mechanical diseases (such as coronary artery diseases).

\section{Conclusion}

The proposed regression model provides a reliable estimation of the electrocardiographic QT interval from phonocardiographic heart sounds in healthy subjects.

\section{References}

[1] A. Subahi, A. S. Yassin, O. Adegbala, M. Shokr, and L. Afonso, "Does a short QT interval increase the risk of cardiac death in healthy people?," Cleve. Clin. J. Med., vol. 87, no. 7, pp. 398-400, Jul, 2020.

[2] G. L. Xiong, A. Pinkhasov, J. P. Mangal, H. Huang, J. Rado, J. Gagliardi, D. Demoss, D. Karol, S. Suo, M. Lang, M. Stern, E. V. Spearman, J. Onate, A. Annamalai, Z. Saliba, T. Heinrich, and J. G. Fiedorowicz, "QTc monitoring in adults with medical and psychiatric comorbidities: Expert consensus from the association of medicine and psychiatry," J. Psychosom. Res, vol. 135, no. 110138, Aug, 2020.

[3] A. Agostinelli, M. Morettini, A. Sbrollini, E. Maranesi, L. Migliorelli, F. Di Nardo, S. Fioretti, and L. Burattini, "CaRiSMA 1.0: Cardiac risk self-monitoring assessment," Open Sports Sci. J., vol. 10, pp. 179-190, Oct, 2017.

[4] R. E. Klabunde, "Cardiovascular Physiology Concepts," Lippincott Williams \& Wilkins, 2004.

[5] C. Giuliani, A. Agostinelli, F. Di Nardo, S. Fioretti, and L. Burattini, "Automatic identification of the repolarization endpoint by computing the dominant T-wave on a reduced number of leads," Open Biomed Eng. J., vol. 10, pp. 43-50, Apr, 2016.

[6] D. Marzorati, D. Bovio, C. Salito, L. Mainardi, and P. Cerveri, "Chest wearable apparatus for cuffless continuous blood pressure measurements based on PPG and PCG signals," IEEE Access, vol. 8, pp. 55424-55437, Mar, 2020.

[7] A. Sbrollini, M. Beghella Bartoli, A. Agostinelli, M. Morettini, F. Di Nardo, S. Fioretti, and L. Burattini, "Second heart sound onset to identify T-wave offset," Comput.
Cardiol., vol. 44, pp. 1-4, Sep, 2017.

[8] P. K. Jain, and A. K. Tiwari, "Heart monitoring systems-A review," Comput. Biol. Med., vol. 54, pp. 1-13, Nov, 2014.

[9] C. Liu, D. Springer, Q. Li, B. Moody, and R. A. Juan, F. J. Chorro, F. Castells, J. M. Roig, I. Silva, A. E. Johnson, Z. Syed, S. E. Schmidt, C. D. Papadaniil, L. Hadjileontiadis, H. Naseri, A. Moukadem, A. Dieterlen, C. Brandt, H. Tang, M Samieinasab, M. R. Samieinasab, R. Sameni, R. G. Mark, and G. D. Clifford, "An open access database for the evaluation of heart sound algorithms," Physiol. Meas., vol. 37, no. 12, pp. 2181-2213, Dec, 2016.

[10] A. L. Goldberger, L. A. Amaral, L. Glass, J. M. Hausdorff, P. C. Ivanov, R. G. Mark, J. E. Mietus, G. B. Moody, C. K. Peng, and H. E. Stanley, "PhysioBank, PhysioToolkit, and PhysioNet: components of a new research resource for complex physiologic signals," Circulation, vol. 101, no. 23 , pp. e215-e220, Month, 2000.

[11] J. Pan, and W. J. Tompkins, "A real-time QRS detection algorithm," IEEE Trans. Biomed. Eng., vol. 32, no. 3, pp. 230-236, Mar, 1985.

[12] P. Laguna, N. V. Thakor, P. Caminal, R. Jané, H. R. Yoon, A. Bayés de Luna, V. Marti, and J. Guindo, "New algorithm for QT interval analysis in 24-hour Holter ECG: performance and applications," Med. Biol. Eng. Comput., vol. 28, no. 1, pp. 67-73, Jan, 1990.

[13] M. Morettini, F. Di Nardo, L. Burattini, S. Fioretti, C. Göbl, A. Kautzky-Willer, G. Panici, and A. Tura, "Assessment of glucose effectiveness from short IVGTT in individuals with different degrees of glucose tolerance," Acta Diabetologica, vol. 55, no. 10, pp. 1011-1018, Jun, 2018.

[14]E. Pietrzyk, Z. Sadowski, H. Szwed, and A. Kraska, "Prognostic value of QT-QS2 index in the assessment of sudden coronary death," Kardiol. Pol., vol. 33, no. 4, pp. 240-249, Apr, 1990.

[15] I. Assmann, P. Kassel, E. Kretzschmar, and I. Porstmann, "Behaviour of the electrical systole (QT) and the ratio of electromechanical systole (QS2)/QT in healthy persons and in patients with cardiac diseases at rest, under drug influence, and heart rate increasing," Electrocardiology, pp. 57-60, Aug, 1988.

[16] H. Boudoulas, C. A. Bush, S. F. Schall, C. V. Leier, and R. P. Lewis, "Prolonged electrical systole and QT > QS2 secondary to coronary artery disease," Am. J. Cardiol., vol. 55, no. 8, pp. 915-919, Apr, 1985.

[17] H. Boudoulas, Y. H. Sohn, W. O'neill, R. Brown, and A. M. Weissler, "The QT > QS2 syndrome: A new mortality risk indicator in coronary artery disease," Am. J. Cardiol., vol. 50, no. 6, pp. 1229-1235, Dec, 1982.

Address for correspondence.

Laura Burattini

Department of Information Engineering,

Università Politecnica delle Marche,

via Brecce Bianche 12,

60131, Ancona, Italy.

E-mail address. 1.burattini@univpm.it 